\title{
A New Watermarking Algorithm for Chaotic Sequence Based On Wavelet
}

\section{Transform}

\section{Zhi-Feng YU ${ }^{1, a}$,Dong ZHONG ${ }^{1, b,{ }^{*}}$, Chun $\mathrm{CHEN}^{2, \mathrm{c}}$ and Ruolin RUAN ${ }^{3, \mathrm{~d}}$ \\ ${ }^{1}$ College of electronic and information, Hubei University of Science and Technology,} Xianning, China

\author{
${ }^{2}$ College of physical education, Hubei University of Science and Technology, Xianning, China; \\ ${ }^{3}$ College of biomedical engineering, Hubei University of Science and Technology, Xianning, China; \\ a27820424@qq.com, bzhongdong0129@163.com, '906572183@qq.com, d251824144@qq.com
}

Keywords: Wavelet transformation, Chaotic sequence, Watermarking algorithm

\begin{abstract}
In this paper, we propose a new watermarking algorithm for chaotic sequence based on wavelet transformation from the traditional digital watermarking algorithm in wavelet domain which has been introduced. We have researched the basic steps of watermarking embedding and extraction algorithms for the chaotic sequence and we have analyzed the new algorithm by the simulation experiments. The results show that the algorithm is adaptive highly. Moreover, it not only could get a better embedding effects, but also it can adjust the depth of the embedding and can be applied to different gray-scale image widely.
\end{abstract}

\section{Introduction}

With the rapid development of the computer network technology, the watermarking algorithm becomes more and more outstanding, which has solved many information obstacles. It becomes a good way to ensure the integrity and reliability of digital information through the detection and analysis of the watermark. The watermarking algorithm also becomes effective means to the protection of intellectual property rights and digital multimedia security ${ }^{[1]}$. It also has been used in multimedia information security, image processing and analysis and other fields. Almost all the previous watermarking algorithms are independent ${ }^{[2]}{ }^{[3]}$.This paper presents a new watermarking algorithm for chaotic sequence based on wavelet transformation. The author studies the basic steps of watermarking embedding and extraction algorithms for the chaotic sequence and analyzes the new algorithm through the simulation experiments. The results show that the algorithm is highly adaptive and get better embedding effects. We found that it also can adjust the depth of the embedding and can be applied to different gray-scale image widely. Experiments show that this algorithm has more practical value.

\section{The wavelet transform watermark algorithm}

The DCT transform is used in the traditional Watermarking algorithm based on transform domain. Due to the wavelet transform is more suitable for human visual system than the DCT transform in image processing (HVS), at the present stage of watermarking algorithm based on wavelet transform has become the study focus of transform domain watermarking algorithm. And 
using DWT (Discrete Wavelet Transform) in JPEG2000 makes the watermarking algorithm based on wavelet transform is more feasible. In wavelet domain, the high frequency part represents the edge and texture image portion. Embedding watermark in here, it is not easy to be found, but such watermarking may be loss after the image had been processed. On the other hand, the low frequency part concentrates most the energy of the image, so the low frequency part has concentrated most information of the image. The power in the low frequency part is not easy to be lost, however, the low frequency part represents the image smooth areas and this part of the change is easy to affect the quality of the image.

The watermarking algorithm based on wavelet transform used in this paper is as follows: firstly, we divided the image, which has been through three level wavelet transform into identical parts with the watermark image block size and without overlap. Then the same watermark had been embedded to each image block. The image area of the high frequency part is larger and it is divided into many small images, so the more of the image block number, the more of the embedded watermark. But the low frequency part accounts for small parts of the image, so the image block quantity are few, the embedded watermark is less naturally. In this way, we can realize the watermark information embedded in the high frequency part is more and the watermark information embedded in the low frequency part is less requirements. The same watermark is repeatedly embedded in different parts of the image, so it is greatly improve the robustness of watermarking.

\section{Application of chaotic sequence in the watermarking algorithm}

Chaotic function has the property of stretch lengthen and retrace overlap, so it is unpredictable. Chaotic sequence is a pseudo-random sequence, which is generated easily by iterative equations or nonlinear equations. In this paper, chaotic sequence generated by discrete time dynamic system Logistic mapping, which is defined as

$$
x_{k+1}=\mu x_{k}\left(1-x_{k}\right), \quad x_{k} \in(0,1)
$$

The bifurcation parameter in $0 \leq \mu \leq 4$ in equation (1), after transformation, the Logistic mapping in $[-1,1]$ can be expressed as

$$
x_{k+1}=1-\lambda x_{k}^{2}, \quad \lambda \in(0,2)
$$

With the $\lambda$ gradual increase, the iteration occur multiple times mutation, when $\lambda=1.40115$, the system enters a chaotic state, then 0 mean, $\delta$ - like self correlation and cross-correlation is 0 , it become chaotic sequence, the statistical properties of the sequence is the same as white noise. This paper takes $\lambda=2$, the initial value $x_{0}=0.3$, according to formula (2) iteration, we obtain a sequence $X_{K}$. The method of chaotic sequences added in digital watermarking has many kinds, the paper adopts the watermarking image $\mathrm{W}$ is expressed as a vector of the form $W_{p}$, $p=1,2, \cdots, M \times N$ 


\section{The basic steps of chaos sequence watermarking embedding and extraction algorithm}

\section{Chaotic Sequence Watermark Embedding Algorithm}

In this paper, watermarking embedding algorithm for chaotic sequence based on wavelet transformation is divided into the following four steps:

(1) Making the original fingerprint image to happen three levels wavelet decomposition, selecting HL3 areas as part of the embedded watermark. In wavelet decomposition, wavelet selection is very important, improper selection will cause algorithm difficult or even fail to realize the consequences. The algorithm in the paper ultimately selected Daubechies9/7 wavelet ${ }^{[4]}{ }^{[5]}$.

(2) There are many ways to embed the chaotic sequence to the watermarking, we use the watermark image $\mathrm{W}$ is expressed as vector form ${ }^{W_{p}}, p=1,2, \cdots, M \times N$.

(3) In general, it is better for low frequency LL embedded in the formula:

$\mathrm{F}^{\prime}(\mathrm{u}, \mathrm{v})=\mathrm{F}(\mathrm{u}, \mathrm{v})(1+\alpha \mathrm{w})$

but it can lead easily to slop over coefficient range when the wavelet is reconstructing in the HL high-frequency region. Therefore, using the following formula :

$$
\mathrm{F}^{\prime}(\mathrm{u}, \mathrm{v})=\mathrm{F}(\mathrm{u}, \mathrm{v})+\alpha \mathrm{w}
$$

$\mathrm{F}(\mathrm{u}, \mathrm{v})$ is the wavelet coefficients of host image, $\alpha$ is the stretch factor. To make the algorithm can apply to different gray-scale images, taking $\alpha=\beta|\mathrm{m}|$, where $\mathrm{m}$ is the mean of all coefficients for watermark embedding part, $\beta$ is the correction factor, representing embedded depth [6].

(4) Finally, there is a wavelet reconstruction for the watermarked image to form a watermarked image after wavelet processing.

\section{Watermark Extraction Algorithm Chaotic Sequence}

Watermark extraction algorithm is the reverse process of embedding algorithm, it requires the original image. Specific steps are as follows:

Making test image to happen three levels wavelet decomposition, then it can obtain the wavelet coefficients $F^{\prime}$ in the HL area, setting an appropriate threshold T, then comparing $F^{\prime}$ with wavelet coefficients $\mathrm{F}$ of the host image in the $\mathrm{HL}_{3},\left|\mathrm{~F}^{-} \mathrm{F}^{\prime}\right| \div \alpha<\mathrm{T}$, If $\left|\mathrm{F}^{\mathrm{F}}\right| \div \alpha<\mathrm{T}$, then the value of taking a watermark to be detected, otherwise, take 0 . Thus, we can get the watermark sequence to be detected, combined with the original watermark sequence $\mathrm{w}$, the probability of hypothesis testing theory, based on the similarity using the following formula:

$$
\rho=\frac{\sum_{i=1}^{N} w(i) w(i){ }^{\prime}}{\sqrt{\sum_{i=1}^{N}\left[w^{\prime}(i)\right]^{2}}}
$$

In the equation(3), $\mathrm{N}$ is expressed as watermark sequence length. Setting a suitable threshold $\rho$ *, if $\rho$ is greater for than $\rho^{*}$, we get the conclusion that the detection image contains the watermark. In general, taking $\rho *=5$, the false positive probability is Gaussian random variable, so the probability of false positives is only sufficient to $\frac{1}{2 \pi} \int_{5}^{\infty} e^{-\frac{t^{2}}{2}} d t \approx 1 \times 10^{-5}$, it can meet the determined 
requirements.

\section{The Characteristics of the Algorithm}

Compared with other digital watermarking algorithm in wavelet domain, the new algorithm, which is proposed in this paper, has two characteristics:

(1) Because the selection of a stretch factor is based on the mean $m$ of the wavelet coefficients, the algorithm is adaptive, it can be widely applied to different gray images.

(2) It can adjust the depth of embedding by adjusting the correction factor $\beta$, to get the best compromise between invisible and robust.

\section{Experimental simulation}

In the experimental simulation, in order to verify the validity of the algorithm to evaluate image quality, the definition of the peak signal to noise ratio:

$$
P S N R=10 \log _{10} \frac{255^{2}}{\left.\frac{1}{N \times N} \sum_{i=1}^{N} \sum_{j=1}^{N} f(i, j)-f^{\prime}(i, j)\right]^{2}}
$$

$\mathrm{N}$ is the image size, $f(i, j)$ and $f^{\prime}(i, j)$ are respectively to expressed the original image and the image to be detected. The value of PSNR is higher, it indicates that the quality of the image to be detected is better than the original image. PSNR $\geq 48 \mathrm{~dB}$ represents that the quality of image is very high, there is no feeling of interference; values of PSNR is good between $35 \mathrm{~dB}$ and $48 \mathrm{~dB}$, and $29 \mathrm{~dB}$ $\sim 35 \mathrm{~dB}$ is the acceptable range, the critical point is $25 \mathrm{~dB}$, lower than the critical value, indicating that the image has generated significant nuisance interference. This paper selects four standard Lena images as the original host images, there are similar results for the other images, taking $\beta=10, \mathrm{~T}=$ 0.5. Comparing the effects of watermark embedding with no watermark embedding, they have been shown in the following 4 sets of images. This is shown in Figure1.

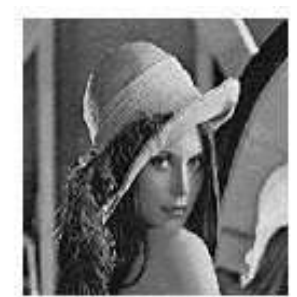

(a) Original Lena Image

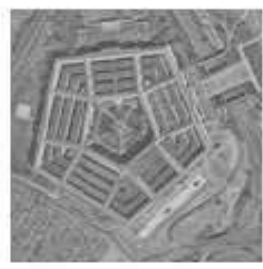

(a) Original Remote Sensing Imag

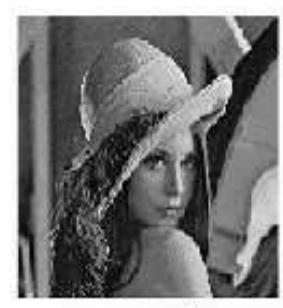

(b) Image with watermark (PSNR $=55.35 \mathrm{~dB}$ )

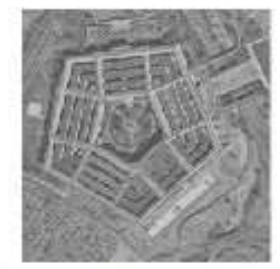

(b)Image with watermark (PSNR=44.70dB)

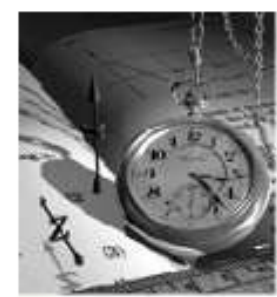

(a) Original Table Image

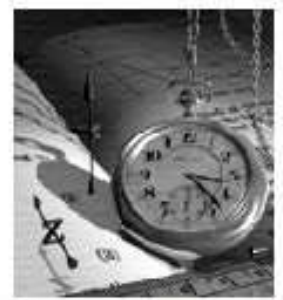

(b)Image with watermark (PSNR=39.08)

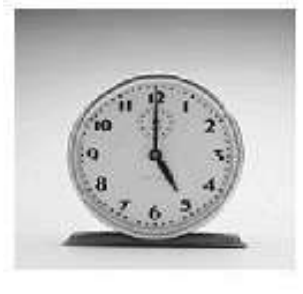

(a) Original Clock Image

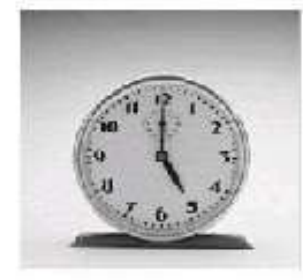

(b)Image with Watermark (PSNR $=59.01 \mathrm{~dB}$ )

Figure1. Original Image and Image with Watermark

We can see that the new watermarking algorithm has a very good effect for the four images. 
Experiment shows that the other has a similar effect of gray image. One main reason is that the choice of stretch factor is based on a mean $m$ of wavelet coefficients, so the algorithm is adaptive. At the same time, the watermark image and original image are almost no difference by the sight of human, and their values of PSNR are greater than $35 \mathrm{~dB}$, that the watermarking embedding algorithm is successful to achieve invisibility of watermarking embedding. If the host images are need to enhance the stealth nature (such as the PSNR up to $48 \mathrm{~dB}$ or more), you need to adjust the correction factor $\beta$, reducing the embedding depth. The experiments selected four kind operations of image processing (adding noise, filtering, wavelet compression of 60magnification, $32 \times 32$ block shear) to attack the watermark image, the results is shown in Table 1.

Analyzing Table1, the value of PSNR decreased significantly after the attacks, and some are down to the critical point $25 \mathrm{~dB}$. it shows that the quality of the image has been destructed, but the correlation coefficient $\rho$ is kept in the fundamental orders of 103 magnitude, it is much larger than the discrimination threshold $\rho *=5$, the algorithm has strong robustness. In addition, $\rho$ is not only related with the attack (type, intensity, etc.), but also related with host images on their own. Table image $\rho$ values, such as 104 orders of magnitude, the effect of anti-attack is high and Clock image is low.

\section{Summary}

This paper presents a new watermarking algorithm for chaotic sequence based on wavelet transform. The algorithm embedded watermarking in the HL sub-graph of three level wavelet transformation, the algorithm achieves not only the invisibility of watermark, but also has a strong robustness for the common image processing. Since the embedded watermark is pseudo-random binary sequence, it can be easily extended to a binary image, which is embedded the adaptive algorithm of grayscale image, and experiments show that the algorithm is a practical digital watermarking algorithm, which can be used widely in the field of information security.

Table 1: The rate of PSNR and Correlation value after attack

\begin{tabular}{|c|c|c|c|c|c|c|c|c|}
\hline \multirow{2}{*}{$\begin{array}{l}\text { Image } \\
\text { attack }\end{array}$} & \multicolumn{2}{|c|}{ Lena Image } & \multicolumn{2}{|c|}{ Clock Image } & \multicolumn{2}{|c|}{$\begin{array}{l}\text { Remote sensing } \\
\text { Image }\end{array}$} & \multicolumn{2}{|c|}{ Table Image } \\
\hline & $\begin{array}{l}\text { PSN } \\
\text { R } \\
(\mathrm{db})\end{array}$ & $\begin{array}{c}\rho \\
\left(* 10^{3}\right) \\
\end{array}$ & $\begin{array}{l}\text { PSNR } \\
\text { (db) }\end{array}$ & $\rho\left(* 10^{3}\right)$ & $\begin{array}{l}\text { PSNR(db } \\
\text { ) }\end{array}$ & $\rho\left({ }^{*} 10^{3}\right)$ & $\begin{array}{l}\text { PSNR(db } \\
\text { ) }\end{array}$ & $\rho\left(* 10^{3}\right)$ \\
\hline $\begin{array}{r}\text { With } \\
\text { Gaussian } \\
\text { noise }\end{array}$ & 23.71 & 1.97 & 24.23 & 1.40 & 24.00 & 3.13 & 24.09 & 11.1 \\
\hline $\begin{array}{l}\text { Winner } \\
\text { filtered }\end{array}$ & 29.65 & 1.97 & 32.57 & 1.71 & 21.86 & 2.45 & 29.68 & 10.4 \\
\hline $\begin{array}{c}60 \\
\text { wavelet } \\
\text { compressed }\end{array}$ & 24.19 & 1.94 & 26.71 & 1.65 & 18.17 & 2.92 & 24.63 & 12.1 \\
\hline $\begin{array}{l}32 \times 32 \\
\text { patches }\end{array}$ & 28.72 & 1.47 & 18.80 & 0.85 & 23.55 & 1.87 & 25.27 & 10.9 \\
\hline
\end{tabular}




\section{Acknowledgement}

The authors gratefully acknowledge the financial support for this work provided by the colleges and universities of Hubei Province in 2014 the Provincial College Students' innovation and entrepreneurship training plan, No. 201410927025 and 201410927029, the National Natural Science Foundation of China (NSFC) under the Grant No. 61271256, the Team Plans Program of the Outstanding Young Science and Technology Innovation of Colleges and Universities in Hubei Province, Grant No.T201513, the Program of the Natural Science Foundation of Hubei Province, Grant No. 2015CFB452, the Scientific research project in Hubei province department of education, Grant No. B2015077.

\section{References}

[1] Bing He, Computer Engineering and Design,30 (1), (2009)145-148.

[2] Longjiang Yu, Sheng Sun, Journal of Electronics,3(12), (2003)2071-2073.

[3] Daren Huang, Jiufen Liu, Software letters, 13 (7), (2002)1290 -1296.

[4] Cui Yingan, Computer Engineering, 22 (33), ( 2007)73-75.

[5] Yun Gu and Dong Chen, Electronic Letters, 38(14), (2002)696-698.

[6] Jianhua Liu, Xiaomei Ma, chengan Guo, The letters of Dalian University of Technology, 42 (5), ( 2002)626-628 